\title{
Size dispersion effects on the low-frequency Raman scattering of quasispherical silver nanoparticles: Experiment and theory
}

\author{
G. Bachelier, ${ }^{1}$ J. Margueritat, ${ }^{2,3}$ A. Mlayah, ${ }^{3}$ J. Gonzalo, ${ }^{2}$ and C. N. Afonso ${ }^{2}$ \\ ${ }^{1}$ Laboratoire de Spectrométrie Ionique et Moléculaire, Université Claude Bernard Lyon 1-CNRS (UMR 5579), 43 Boulevard du 11 \\ Novembre 1918, 69622 Villeurbanne Cedex, France \\ ${ }^{2}$ Laser Processing Group, Instituto de Optica, CSIC, Serrano 121, 28006 Madrid, Spain \\ ${ }^{3}$ Centre d'élaboration de Matériaux et d'Etudes Structurales, CNRS-Université Paul Sabatier, 29 Rue Jeanne Marvig, 31055 Toulouse \\ Cedex 4, France
}

(Received 6 April 2007; revised manuscript received 27 August 2007; published 18 December 2007)

\begin{abstract}
The coupling between surface plasmon polaritons and confined acoustic vibrations in embedded silver nanoparticles is studied both theoretically and experimentally. The inelastic light scattering spectra simulated assuming deformation potential and surface orientation mechanisms are quantitatively compared to Raman measurements. The effects of size distribution (width and profile) and excitation energy on the low-frequency Raman spectra are addressed in this work and used to provide a characterization tool of the size distribution of metal nanoparticles. The quantitative agreement between measured and simulated spectra supports the fact that the surface orientation mechanism gives the dominant contribution to the resonant Raman scattering mediated by surface plasmon polaritons.
\end{abstract}

DOI: 10.1103/PhysRevB.76.235419

PACS number(s): 63.22.+m, 73.22.Lp, 78.30.Er, 78.67.Bf

\section{INTRODUCTION}

When excited optically close to their surface-plasmonpolariton (SPP) resonance, ${ }^{1,2}$ metal nanostructures lead to strong inelastic light scattering due to emission or absorption of vibration modes. This is well known as surface enhanced Raman scattering ${ }^{3}$ for molecules located close to noble metal nanoparticles and can be understood in terms of localization and thus magnification of the electromagnetic field. However, this effect can also be used to evidence the vibration modes of the nanostructures themselves. ${ }^{4}$ Since those vibration modes strongly depend on the nanostructure morphology (size and shape), the coupling between SPPs and vibrations has early been considered as a potential tool for characterization.

The low-frequency Raman scattering involving terahertz vibrations was first investigated by Weitz et al. ${ }^{4}$ on rough metal electrodes. The coupling mechanism was assigned to the modulation of the electronic density, and consequently of the SPP resonance, by vibrations. In the special case of spherical nanoparticles, this coupling mechanism was expected to excite dominantly the quadrupolar vibration modes $(l=2)$. On the contrary, radial modes were supposed to be inefficient. ${ }^{4}$ This was apparently in agreement with many experimental observations. ${ }^{4-6}$ Montagna and Dusi ${ }^{7}$ proposed another mechanism of light scattering based on the dipoleinduced-dipole and bond polarizability models. However, this approach is only suitable for nonresonant scattering in dielectric materials. Thus, the recent observation of new vibration modes, namely, the radial $(l=0)$ modes, in lowfrequency Raman scattering, ${ }^{8,9}$ has raised new interest for both theoretical and experimental studies.

In a previous work, ${ }^{11}$ various coupling mechanisms between surface plasmon polaritons and confined acoustic vibrations were investigated theoretically. It has been shown that the low-frequency Raman spectra are indeed dominated by the contribution of two coupling mechanisms: the defor- mation potential (DP) mechanism due to the modulation of the interband dielectric susceptibility by pure radial vibrations $(l=0)$ and the surface orientation ( $\mathrm{SO})$ mechanism corresponding to the modulation of the surface polarization charges by quadrupolar vibrations $(l=2)$. In this work, we present the first quantitative comparison between experiments and simulations performed using the SO and DP mechanisms. This paper should therefore be considered as a sequel of Ref. 11. Raman spectra of (quasi)spherical silver nanoparticles (NPs) embedded in amorphous $\mathrm{Al}_{2} \mathrm{O}_{3}$ host matrix are measured for both resonant and nonresonant excitations and compared to the simulated spectra, revealing the importance of the size distribution profile and size dispersion. The aim of this work is to show that the dependence of the inhomogeneously broadened Raman lines on the excitation energy allows one to determine the unique couple of mean size and size dispersion, which gives a good agreement between simulated and measured spectra.

\section{EXPERIMENTS}

$\mathrm{Ag}: \mathrm{Al}_{2} \mathrm{O}_{3}$ nanocomposite films containing five layers of $\mathrm{Ag}$ NPs embedded in amorphous $\mathrm{Al}_{2} \mathrm{O}_{3}\left(a-\mathrm{Al}_{2} \mathrm{O}_{3}\right)$ were grown by pulsed laser deposition (PLD) in vacuum $\left(10^{-6}\right.$ mbar $)$. An ArF excimer laser beam $(\lambda=193 \mathrm{~nm}, \tau$ $=20 \mathrm{~ns}$ full width at half maximum) operating at $10 \mathrm{~Hz}$ was alternatively focused on the surface of high purity $\mathrm{Al}_{2} \mathrm{O}_{3}$ and $\mathrm{Ag}$ targets at an angle of incidence of $45^{\circ}$. An average energy density of $\approx 2 \mathrm{~J} \mathrm{~cm}^{-2}$ was used to ablate both targets. PLD produces approximately spherical NPs for low metal contents that evolve to ellipsoids when the metal content is increased due to coalescence. The use of alternate PLD allows precisely controlling the in-depth separation between consecutive layers of NPs, through the number of laser pulses on the $\mathrm{Al}_{2} \mathrm{O}_{3}$ target. Further details of the synthesis procedure as well as the morphological analysis can be found 


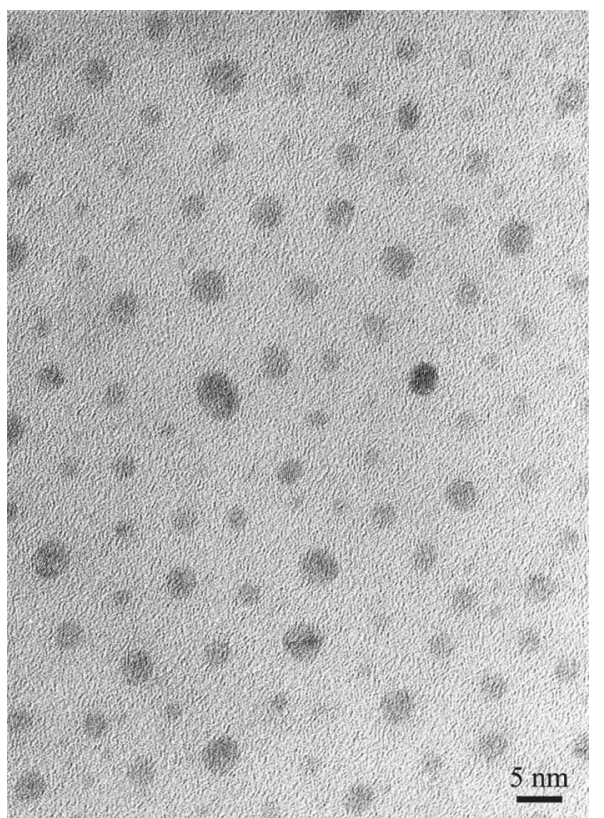

FIG. 1. Plan view HREM image of a sample produced in the same experimental conditions as the studied one.

elsewhere. ${ }^{12,13}$ In an earlier work, films consisting of five layers of Ag NPs with diameters in the range 1-12 nm and in-depth separations between consecutive layers ranging from $\approx 4.6$ to $29.4 \mathrm{~nm}$, while maintaining approximately constant the in-plane (surface to surface) NP separation $(\approx 6 \pm 2 \mathrm{~nm})$, were reported. ${ }^{12}$ For this work, we have selected a specimen having approximately spherical NPs, and the NP layers being surface to surface in depth separated by $21 \pm 2 \mathrm{~nm}$ of $a-\mathrm{Al}_{2} \mathrm{O}_{3}$.

Figure 1 shows a plan view high resolution electron microscopy image of a sample produced in the same experimental conditions as the one studied in this work. The NPs (dark areas) present a quasicircular shape and are randomly distributed in the plane. In order to investigate their in-plane shape and size distribution, this image has been digitized following a procedure described elsewhere. ${ }^{13}$ Table I summarizes the main morphological parameters obtained from this analysis: in-plane average diameter, NP height, NP separation, and NP number density. ${ }^{12}$

The Raman scattering was excited at room temperature using the output lines of argon and krypton lasers. The scattered light was dispersed through a triple spectrometer and detected with a conventional single channel photon counting system.

TABLE I. Morphological parameters obtained from HREM (Refs. 12 and 13) on the studied sample. Note that the in-plane mean separation corresponds to a surface to surface separation.

Areal density (\%)

In-plane average diameter ( $\mathrm{nm}$ )

$2.9 \pm 0.8$

Average height (nm)

$3.2 \pm 0.6$

In-plane mean separation $(\mathrm{nm})$

$6 \pm 2$

Number density of NPs (NPs/ $\left.\mathrm{cm}^{2}\right)$

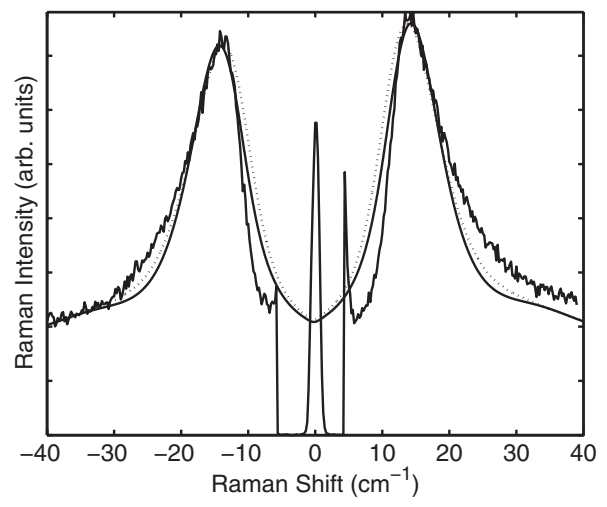

FIG. 2. Measured Raman spectrum of silver particles with $1.4 \mathrm{~nm}$ mean radius excited at $413 \mathrm{~nm}$ (close to the $\mathrm{SPP}$ resonance). The dotted and full lines correspond to simulations performed with a log-normal size distribution using $\langle R\rangle=1.3 \mathrm{~nm}, \sigma=20 \%$ and $\langle R\rangle$ $=1.4 \mathrm{~nm}, \sigma=15 \%$, respectively.

\section{RESULTS AND DISCUSSION}

Figure 2 shows the Stokes and anti-Stokes low-frequency Raman spectrum of the studied specimen excited at $413 \mathrm{~nm}$, close to the SPP resonance. This spectrum is typical from spherical metal NPs. The strong emission (and absorption) band around $14 \mathrm{~cm}^{-1}$ is due to quadrupolar acoustic vibrations $(l=2)$. The low-intensity bands reported by Portales et $a l .{ }^{8}$ and Courty et al. ${ }^{9}$ at higher frequencies are not resolved in this spectrum.

Optical absorption spectra of nanocomposites having $\approx 3 \mathrm{~nm}$ diameter NPs were reported for in-depth separation ranging from 4.6 to $29.4 \mathrm{~nm} .^{12}$ No significant difference was found, showing that electromagnetic interactions between particles, through the near field associated with the SPP states, can be neglected. Note that this was not the case for the NPs coated by organic chains studied by Courty et al. ${ }^{9,14}$ for which the size parameter $(\approx 3.5 / 2.25)$, given by the ratio between the particle distance and the particle radius, was smaller than that in the present case $(\approx 6 / 1.5)$.

Raman spectra were also recorded for in-depth separations down to $4.6 \mathrm{~nm}$ leading to the same conclusion concerning the mechanical properties of the nanoparticles: despite the $a-\mathrm{Al}_{2} \mathrm{O}_{3}$ matrix between the layers of $\mathrm{Ag}$ NPs, no evidence for line broadening or splitting was found, so that the particles can also be considered as mechanically isolated. ${ }^{15}$ This is a key point in order to apply the model developed by Bachelier et al. ${ }^{11}$ accounting for the lowfrequency Raman scattering of isolated metal particles. This model is based on the modulation of the polarization vector $\mathbf{P}_{\omega, L, M}=\chi \mathbf{E}_{\omega, L, M}$ assigned to the SPP state $(\omega, L, M)$ and induced by the vibrations $(n, l, m)$ of the nanoparticles. $L, M, l$, and $m$ are the usual quantum numbers corresponding to the angular momentum $(-L \leqslant M \leqslant L$ and $-l \leqslant m \leqslant l), \hbar \omega$ is the SPP energy, and $n$ labels vibration modes having the same angular momentum but increasing energies. The modulation $\delta_{n, l, m} \mathbf{P}_{\omega, L, M}$ of the polarization vector was found to be either due to a modulation of the interband susceptibility $\chi^{i b}(\omega)$ via the deformation potential (DP mechanism) or to a direct 
modulation of the electric field $\mathbf{E}_{\omega, L, M}$ due to the modification of the particle shape. ${ }^{11}$ The latter mechanism can be analyzed in terms of a change of the surface orientation $\delta_{n, l, m} \mathbf{n}$ due to the particle vibration (SO mechanism) leading to a redistribution of the SPP surface charges. Both mechanisms were evaluated in the following simulations. The pre- cise forms of the polarization vector modulation $\delta_{n, l, m} \mathbf{P}_{\omega, L, M}$ are given in Ref. 11. The vibration modes and their divergence were computed within the frame of the Lamb theory. ${ }^{16}$ Using a dipolar electric Hamiltonian (P.E) to account for each interaction step, the resonant Raman scattering probability is proportional to ${ }^{11}$

$$
\left|\sum_{\omega^{\prime}, L^{\prime}, M^{\prime}} \sum_{\omega, L, M} \frac{\int \mathbf{E}_{s} \cdot \mathbf{P}_{\omega^{\prime}, L^{\prime}, M^{\prime}} d V \int \mathbf{E}_{\omega^{\prime}, L^{\prime}, M^{\prime}} \cdot \delta_{n, l, M} \mathbf{P}_{\omega, L, M} d V \int \mathbf{P}_{\omega, L, M} \cdot \mathbf{E}_{i} d V}{\left(\hbar \omega_{s}-\hbar \omega^{\prime}+i \Gamma_{\omega^{\prime}, L^{\prime}}\right)\left(\hbar \omega_{i}-\hbar \omega+i \Gamma_{\omega, L}\right)}\right|^{2},
$$

where $i$ and $s$ label the incident and scattered photons and $\Gamma_{\omega, L}$ accounts for the size dependent homogeneous broadening of the SPPs due to the Landau damping. ${ }^{10,11}$ Note that the use of dipolar electric Hamiltonians does not restrict the model to the dipolar approximation $(L=1)$, as indicated by the sums over $L$ and $L^{\prime}$.

Comparing the theoretical predictions to the experimental Raman data is essential to check the validity of the model presented above. In order to account for the size distribution of the studied sample, leading to inhomogeneous broadening of the Raman peaks, we use, in a first step, a log-normal distribution,

$$
\rho(R)=\frac{1}{\sqrt{2 \pi} S R} \exp \left\{-\frac{[\ln (R)-M]^{2}}{2 S^{2}}\right\},
$$

where

$$
S=\sqrt{\ln \left(\frac{\sigma^{2}+\langle R\rangle^{2}}{\langle R\rangle^{2}}\right)}, \quad M=\ln \left[\frac{\langle R\rangle}{\exp \left(\frac{S^{2}}{2}\right)}\right],
$$

$R$ being a particle radius, and $\langle R\rangle$ and $\sigma$ the mean radius and size dispersion, respectively. The simulated spectra are shown in Fig. 2, where it is seen that a reasonable agreement with measured Raman spectra can be obtained for various couples of particle mean radius and size dispersion (only two are shown here). It is therefore not possible to determine the actual couple of parameters using only this comparison. A detailed study of the effect of the size dispersion on the lowfrequency Raman spectra is thus needed to precisely analyze the experimental data.

\section{A. Size effects}

In the framework of Lamb theory, the frequency of the acoustic vibration modes is inversely proportional to the particle radius. ${ }^{16}$ For very small size dispersions, this scaling can directly be used to extract the mean particle radius. ${ }^{8,18}$ However, for larger size dispersions, the dependence of the scattered intensity on the particle size has to be taken into account since it can strongly affect the relation between the size distribution maximum and the Raman intensity maximum.

To highlight the effect of the size dispersion on the lowfrequency Raman spectra, four scattering corresponding to log-normal size distributions with $\sigma=5 \%, 10 \%, 20 \%$, and $30 \%$ are shown in Fig. 3. They were calculated by averaging the Raman scattering probability [Eq. (1)] weighted by the size distribution [Eq. (2)]. As one can see, there are two main effects. First is the disappearance of the low-intensity bands in the high-frequency tail of Raman spectra as the size dispersion increases. This effect is due to the size dependence of the vibration frequencies $\Omega_{n, l, m}$ being proportional to $1 / R$. The inhomogeneous broadening due to size dispersion $\sigma$ is proportional to $\sigma \Omega_{n, l, m}^{2}$ (if one neglects the size dependence of the scattering probability). Thus, the higher the vibration frequency, the broader the peak. For size dispersions larger than $20 \%$, only the most intense peak clearly comes out in the simulations. This could explain why the weak Raman bands due to the pure radial modes $(l=0)$ were only recently

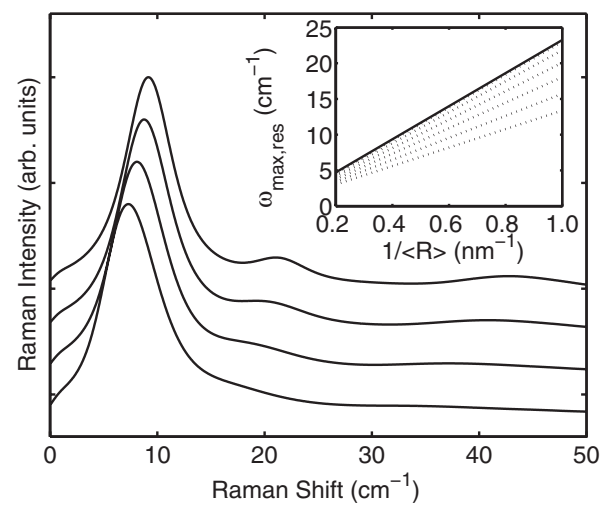

FIG. 3. Normalized Raman spectra calculated with DP $\left(D^{e-p h}\right.$ $=-1.55 \mathrm{eV})$ (Ref. 17) and SO mechanisms for a mean particle radius of $2.5 \mathrm{~nm}$. The size dispersions, corresponding to a log-normal distribution, are equal to $5 \%, 10 \%, 20 \%$, and $30 \%$ from top to bottom. The inset shows the frequency in $\mathrm{cm}^{-1}$ of the main Raman peak as a function of the inverse particle mean radius for size dispersions of $0 \%, 5 \%, 10 \%, 20 \%, 30 \%, 40 \%$, and $50 \%$ from top to bottom. 


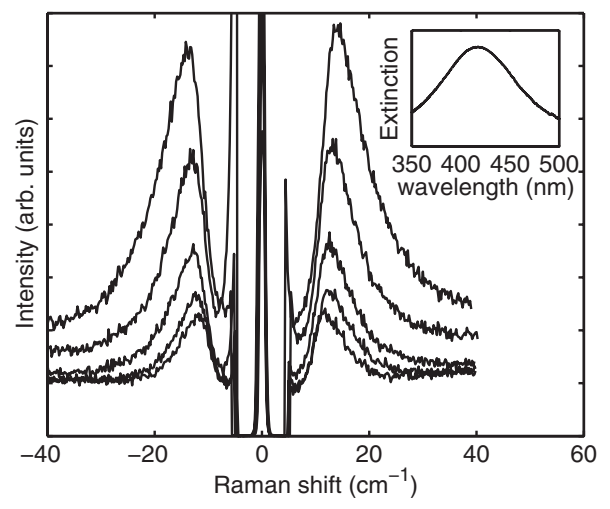

FIG. 4. Raman spectra measured on silver particles with $1.4 \mathrm{~nm}$ mean radius and excited at 413, 458, 476, 488, and $514 \mathrm{~nm}$ from top to bottom. The corresponding extinction spectrum showing the SPP resonance is plotted in the inset.

observed on silver NPs with very small size dispersion. ${ }^{8}$

The second effect is related to the shift of the main Raman peak toward low frequencies as the size dispersion increases. This is a direct consequence of the size dependence of the scattering probability, mainly due to the enhancement of the electric field $\mathbf{E}_{\omega, L, M}$ induced by its localization around the NPs and to the confinement of vibrations inside the particles. ${ }^{15,19}$ Both effects are responsible for the size dependent amplitude of the eigenstates $(\omega, L, M)$ and $(n, l, m)$. More precisely, the SPP electric field was found to be inversely proportional to the particle volume at the SPP resonance and the vibration amplitude to scale as $1 / R^{2}$.

It is interesting to note, as shown in the inset of Fig. 3, that the intensity maximum still shifts linearly with the inverse of the mean particle radius for a given size dispersion. The slope of $\Omega_{\max }(1 /\langle R\rangle)$ depends thus on the size dispersion. This underlines how much the particle radius can be overestimated by a direct comparison between the frequency of the Raman intensity maximum and the calculated vibration mode frequency.

\section{B. Resonance effects}

The purpose of this section is to show how to extract both the mean size and the size dispersion of the nanoparticles from the Raman spectra. For this purpose, we use the resonance effects evidenced by Portales et al..$^{8}$ the Raman intensity maximum shifts toward low frequencies for decreasing excitation energies, as shown in Fig. 4, for the sample studied in this work. This behavior was recently analyzed in terms of size dependence of the scattering probability. ${ }^{15}$ More precisely, normalizing the SPP with respect to their far-field component, the electric field out of resonance was found to be nearly independent of the particle radius, in contrast with the $1 / V$ scaling close to resonance. Moreover, special care must be paid while deriving the size dependence of the scattering probability due to the sums over the SPP states [see Eq. (1)]. Indeed, in the model proposed in Ref. 15 the "number" of SPP states involved as intermediate states of the light scattering is proportional to the volume for resonant

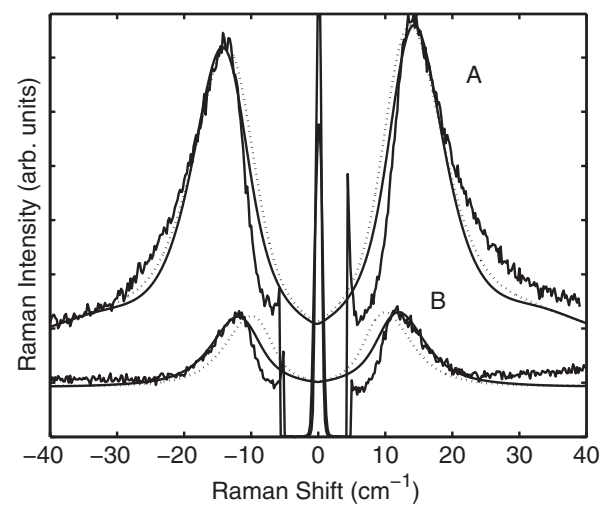

FIG. 5. Comparison between measured and simulated Raman spectra (A) in and (B) out of the SPP resonance. The simulations were performed with mean radii and size dispersions (log-normal distribution) of $\langle R\rangle=1.3 \mathrm{~nm}, \sigma=20 \%$ (dotted line) and $\langle R\rangle$ $=1.4 \mathrm{~nm}, \sigma=15 \%$ (full line).

excitation (width of the resonance), whereas it is independent of the particle size out of resonance. Finally, replacing each term of Eq. (1) by its specific size dependence (the electric field of the incident and scattered photons being independent of $R$ ), the scattering probability was found to scale as

$$
\left|V V\left(1 \frac{1}{V} V\right)\left(\frac{1}{V} \frac{1}{R^{2}} \frac{1}{V} V\right)\left(\frac{1}{V} 1 V\right)\right|^{2}=R^{2}
$$

at the SPP resonance and

$$
\left|(1 \cdot 1 V)\left(1 \frac{1}{R^{2}} 1 V\right)(1 \cdot 1 V)\right|^{2}=V^{5} / R
$$

below the SPP resonance. ${ }^{11}$ Multiplying by the Bose-Einstein factor associated with the low-frequency vibrations, which introduces an additional term proportional to the particle radius $R$, one obtains a Raman intensity proportional to $V$ for an excitation at the SPP resonance and to $V^{5}$ for nonresonant excitation. ${ }^{15,22}$ As a consequence, and for a given size dispersion, the shift of the Raman peak toward low frequencies, with respect to the case of monodisperse particles, is larger for excitation at energies below the SPP resonance than for the resonant excitation.

The two couples of mean radii and size dispersions $(\langle R\rangle, \sigma)$ used in Fig. 2, and giving a reasonable agreement with experiments for an excitation at the SPP resonance, can now be discriminated, thanks to the nonresonant Raman spectra. As one can see in Fig. 5, the shift of the Raman peak for the nonresonant excitation depends on the couple of parameters $(\langle R\rangle, \sigma)$, so that one can rule out the case of $\langle R\rangle$ $=1.3 \mathrm{~nm}$ and $\sigma=20 \%$ and retain the values $\langle R\rangle=1.4 \mathrm{~nm}$ and $\sigma=15 \%$, giving a better agreement for both resonant and nonresonant excitations.

At this step, one can propose a reliable tool to extract the mean radius and size dispersion $(\langle R\rangle, \sigma)$ by plotting the calculated frequency $\Omega_{\text {max,res }}$ of the intensity maximum for resonant excitation as a function of the frequency $\Omega_{\text {max, nonres }}$ of the Raman intensity maximum for nonresonant excitation (see Fig. 6). The line splitting the graph into two equal parts 


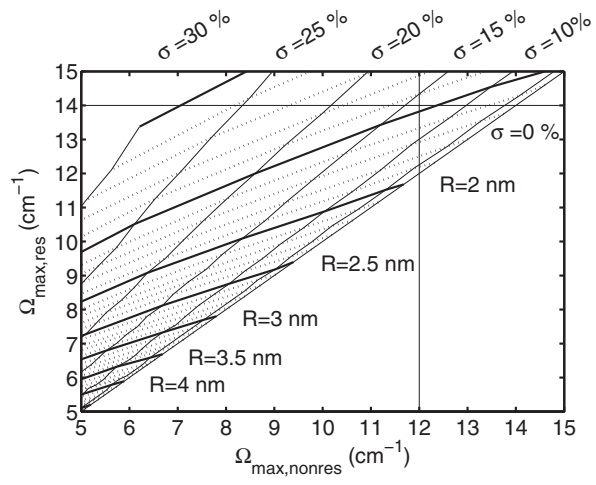

FIG. 6. ABACUS giving the in resonance versus out of resonance frequency $\left(\right.$ in $\mathrm{cm}^{-1}$ ) of the Raman intensity maximum. The full and dotted bold lines correspond to increasing particle radius as indicated. The thin line splitting the graph into two equal parts corresponds to a log-normal size distribution with $\sigma=0 \%$ (monodisperse). From right to left, the size dispersion increases from $0 \%$ to $30 \%$ with an increment of 5\% (thin lines). The case of the studied sample is also reported as the crossing point between the horizontal and vertical thin lines $\left(\Omega_{\text {max, res }}=14 \mathrm{~cm}^{-1}\right.$ and $\left.\Omega_{\text {max, nonres }}=12 \mathrm{~cm}^{-1}\right)$.

corresponds to a monodisperse distribution (there is no downward shift whatever the excitation energy). From the measured spectra shown in Fig. 5, we found $\Omega_{\text {max }}$,es $=14 \mathrm{~cm}^{-1}$ and $\Omega_{\text {max,nonres }}=12 \mathrm{~cm}^{-1}$, giving a mean particle radius of $1.5 \pm 1 \mathrm{~nm}$ and a size dispersion of $(13 \pm 2) \%$ (assuming an uncertainty on the intensity maximum position of $\left.\approx 1 \mathrm{~cm}^{-1}\right)$. This determination, based only on the measured frequencies of the Raman intensity maxima, is in good agreement with the results obtained previously by fully analyzing the Raman line shape, as discussed in Fig. 5.

It is interesting to notice that the size dispersion deduced from the comparison between the Raman measurements and the simulations is almost half than that determined by HREM $(\approx 28 \%)$, cf. Table I. In order to determine the origin of this discrepancy, we analyzed in detail the size distribution from the plan view HREM image (Fig. 7). As a matter of fact, the size distribution presents a double peak fitted by log-normal distributions with average radii of $1.05 \pm 0.2$ and $1.7 \pm 0.2 \mathrm{~nm}$

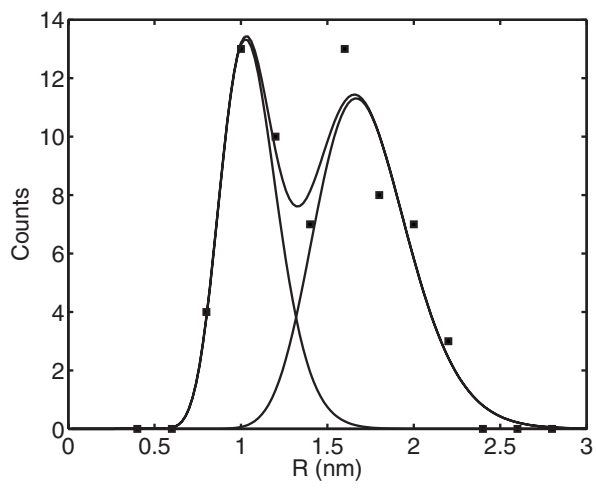

FIG. 7. Size distribution (squares) deduced from the plan view HREM image (Fig. 1). The lines correspond to log-normal distributions with average radii of 1.05 and $1.7 \mathrm{~nm}$ and size dispersion of $15 \%$ for each distribution.

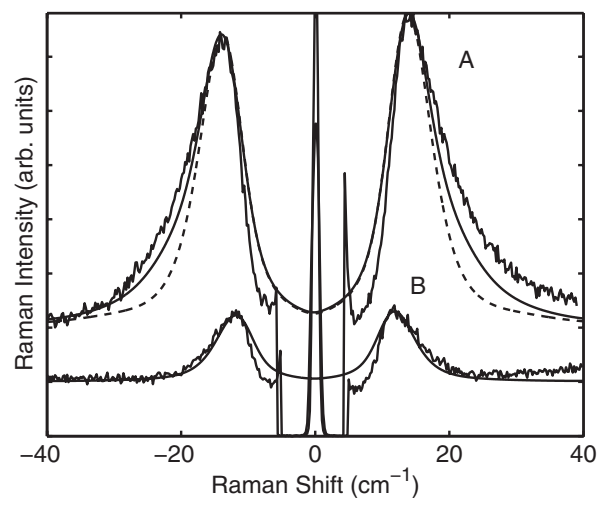

FIG. 8. Comparison between measured and simulated Raman spectra (A) close to and (B) below the SPP resonance. The dashed and full lines correspond, respectively, to mono- and bimodal size distributions (see text).

and a size dispersion of $15 \%$ in both cases. The latter value is in close agreement with our simulations. On the other hand, the bimodal distribution necessarily has a corresponding signature on the low-frequency Raman spectra. Due to the $1 / R$ dependence of the vibration frequencies, small particles contribute to the Raman spectra at higher frequencies than the large particles. Moreover, according to the size dependence of the Raman scattering efficiency (proportional to $V$ for resonant excitation), the expected contribution of small particles is weaker than that of large particles, leading finally to the shoulder, around $25 \mathrm{~cm}^{-1}$, in the high-frequency part of the principal peak [Figs. 2 and 5(A)]. For excitation below the SPP resonance, the size dependence of the scattering cross section is sharper $\left(V^{5}\right)$ so that the small particle contribution completely vanishes: the shoulder visible in Fig. 5(A) disappeared in Fig. 5(B). To corroborate this qualitative agreement, we simulate the Raman spectra using a monomodal distribution $(\langle R\rangle=1.5 \mathrm{~nm}, \sigma=13 \%)$ and a bimodal distribution $(\langle R\rangle=1.5 \mathrm{~nm}, \sigma=13 \%$ and $\langle R\rangle=1 \mathrm{~nm}, \sigma=13 \%)$, cf. the dashed and full lines in Fig. 8, respectively. The relative amplitude of the two distributions was chosen to be equal to 1.4 in the simulations in close agreement with the

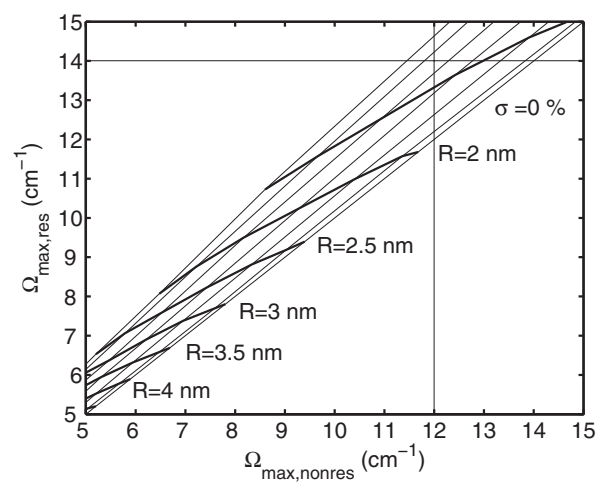

FIG. 9. Same plots as in Fig. 6 with a Gaussian size distribution profile. 
value deduced from the size histogram shown in Fig. 7 $(7.6 / 5.5 \approx 1.38)$. One can clearly see that a better agreement between measured and simulated spectra is obtained with the bi-modal size distribution (Fig. 8). This not only underlines the sensitivity of the low-frequency Raman scattering to the details of the particle size distribution but also corroborates the interpretation of the light scattering process in terms of the surface orientation mechanism.

We would like to stress here the high sensitivity of the simulations to the size distribution profile. For instance, we reproduce in Fig. 9 the same calculations as in Fig. 6 but with a Gaussian size distribution (truncated to the positive values of the particle radius) instead of the log-normal size distribution. The main effect is to modify the proportion of large and small particles for a given mean radius and size dispersion $(\langle R\rangle, \sigma)$. As one can see in Fig. 9, the results strongly differ from those obtained with a log-normal distribution for size dispersions higher than $10 \%$. Hence, special attention must be paid, while comparing measured Raman data and simulations, to the specific size distribution of the studied sample.

\section{CONCLUSION}

The model based on surface orientation and potential deformation mechanisms has been used to simulate the Raman scattering from quasispherical and noninteracting silver nanoparticles. The calculated Raman spectra were successfully compared to the measured spectra. In particular, the size dependence of the scattering efficiency is well reproduced for both resonant and nonresonant excitations. The main conclusion of this work is that, for size dispersion higher than $10 \%$, the size distribution profile plays an important role. It must be fully taken into account in the simulation in order to accurately correlate the frequency of the Raman peaks to the nanoparticle mean size. We showed that both resonantly and nonresonantly excited spectra have to be compared to the simulation in order to precisely account for the size distribution of the nanoparticles.

\section{ACKNOWLEDGMENTS}

This work was partially supported by MAT2005-06508C02-01, MEC Spain. J.M. acknowledges financial support from the CSIC and the European Social Fund.
${ }^{1}$ K. Arya and R. Zeyher, in Light Scattering in Solids IV, edited by M. Cardona and G. Güntherodt (Springer-Verlag, Berlin, 1984). ${ }^{2}$ G. Mie, Ann. Phys. 25, 377 (1908).

${ }^{3}$ M. Moscovits, Rev. Mod. Phys. 57, 783 (1985).

${ }^{4}$ D. A. Weitz, T. J. Gramila, A. Z. Genack, and J. I. Gersten, Phys. Rev. Lett. 45, 355 (1980).

${ }^{5}$ M. Fujii, T. Nagareda, S. Hayashi, and K. Yamamoto, Phys. Rev. B 44, 6243 (1991).

${ }^{6}$ B. Palpant, H. Portales, L. Saviot, J. Lerme, B. Prevel, M. Pellarin, E. Duval, A. Perez, and M. Broyer, Phys. Rev. B 60, 17107 (1999); E. Duval, H. Portales, L. Saviot, M. Fujii, K. Sumitomo, and S. Hayashi, ibid. 63, 075405 (2001).

${ }^{7}$ M. Montagna and R. Dusi, Phys. Rev. B 52, 10080 (1995).

${ }^{8}$ H. Portales, L. Saviot, E. Duval, M. Fujii, S. Hayashi, N. Del Fatti, and F. Vallee, J. Chem. Phys. 115, 3444 (2001).

${ }^{9}$ A. Courty, I. Lisiecki, and M. P. Pileni, J. Chem. Phys. 116, 8074 (2002).

${ }^{10}$ R. A. Molina, D. Weinmann, and R. A. Jalabert, Phys. Rev. B 65 , 155427 (2002).

${ }^{11}$ G. Bachelier and A. Mlayah, Phys. Rev. B 69, 205408 (2004).

${ }^{12}$ J. Gonzalo, R. Serna, J. Solís, D. Babonneau, and C. N. Afonso, J. Phys.: Condens. Matter 15, 3001 (2003).

${ }^{13}$ J.-P. Barnes, A. K. Petford-Long, R. C. Doole, R. Serna, J. Gonzalo, A. Suárez-García, C. N. Afonso, and D. Hole, Nano- technology 13, 465 (2002).

${ }^{14}$ A. Courty, P. A. Albouy, A. Mermet, E. Duval, and M. P. Pileni, J. Phys. Chem. B 109, 21159 (2005).

${ }^{15}$ G. Bachelier, Ph.D. thesis, P. Sabatier University, 2004.

${ }^{16}$ A. E. H. Love, A Treatise on the Mathematical Theory of Elasticity (Dover, New York, 1944).

${ }^{17}$ The value of $-1.55 \mathrm{eV}$ for the deformation potential was adjusted to give a good agreement with the experimental data of Refs. 8 and 9.

${ }^{18}$ A. Nelet, A. Crut, A. Arbouet, N. Del Fatti, F. Vallée, H. Portales, L. Saviot, and E. Duval, Appl. Surf. Sci. 226, 209 (2004).

${ }^{19}$ The effect of the matrix is accounted for by the introduction of a size dependent homogeneous broadening (Refs. 11 and 20). A more complete description was proposed by Murray and Saviot (Ref. 21) but is beyond the scope of the present work.

${ }^{20}$ N. Del Fatti, C. Voisin, F. Chevy, F. Vallee, and C. Flytzanis, J. Chem. Phys. 110, 11484 (1999).

${ }^{21}$ D. B. Murray and L. Saviot, Phys. Rev. B 69, 094305 (2004); Physica E (Amsterdam) 26, 417 (2005).

${ }^{22}$ Additional size dependences arise from the denominators of the scattering probability, due to the Landau Damping associated with the SPP states (Ref. 11). Such effects together with the homogeneous broadening induced by the matrix on the vibration modes (Ref. 19) were taken into account in the simulations. 